\title{
Fourier-Transform Electrospray Instrumentation for Tandem High-Resolution Mass Spectrometry of Large Molecules
}

\author{
Steven C. Beu, Michael W. Senko, John P. Quinn, Francis M. Wampler III, \\ and Fred W. McLafferty \\ Department of Chemistry, Baker Laboratory, Cornell University, Ithaca, New York, USA
}

\begin{abstract}
Mass spectrometry instrumentation providing unit resolution and 10-ppm mass accuracy for molecules larger than $10 \mathrm{kDa}$ was first reported in 1991 . This instrumentation has now been improved with a 6.2-T magnet replacing that of $2.8 \mathrm{~T}$, a more efficient vacuum system, ion injection with controlled ion kinetic energies, accumulated ion trapping with an opencylindrical ion cell, acquisition of $2 \mathrm{M}$ data points, and updated electrospray apparatus. The resulting capabilities include resolving power of $5 \times 10^{5}$ for a 29-kDa protein, less than 1-ppm mass measuring error, and dissociation of protein molecular ions to produce dozens of fragment ions whose exact masses can be identified from their mass-to-charge ratio values and isotopic peak spacing. (J Am Soc Mass Spectrom 1993, 4, 557-565)
\end{abstract}

$\mathrm{S}$ tructural characterization by mass spectrometry is especially favorable for long-chain molecules, such as proteins, nucleotides, and carbohydrates, because backbone bond dissociation produces fragments whose masses are indicative of the structural units [1-3]. This has become an especially powerful routine method for oligopeptide sequencing $[4,5]$, even at the subpicomole level [6]. Of the 20 common amino acids of peptides/proteins, only leucine and isoleucine have identical masses, whereas the masses of lysine and glutamine differ by only $37 \mathrm{mDa}$. Complex mixtures of oligopeptides $(<3 \mathrm{kDa})$ can be routinely sequenced using a preliminary separation, such as by liquid chromatography, to yield fractions containing only a few oligopeptides. Tandem mass spectrometry (MS/MS, MS ${ }^{n}$ ) can then separate molecular ions produced by soft ionization representing only one component of the mixture, with dissociation of these ions and MS-II analysis of the fragments to provide sequence information for the individual components [4-9]. Further dissociation of such fragment ions (such as MS f MS/MS) can then be used to distinguish Leu/Ile isobars $[4,5,10]$ and provide confirmatory sequence information.

These same principles should apply to the structural characterization of even larger proteins and other long-chain molecules, but this requires a variety of improvements in techniques for ionization and ion dissociation and in instrumentation for resolution and mass measuring accuracy. Of revolutionary impor-

Address correspondence to Fred W. McLafferty, Department of Chemistry, Baker Laboratory, Cornell University, Ithaca, NY 138531301. tance has been the development of several techniques for the ionization of large molecules, such as plasma desorption [11], fast-atom bombardment [12], matrixassisted laser desorption [13], and electrospray ionization (ESI) [14-16]. For example, collisionally activated dissociation of singly charged oligopeptide ions has an upper limit of approximately $3 \mathrm{kDa}[4,5,17]$; ESI provides a very promising solution in that there appears to be no upper mass limit to the collisional dissociation of the multiply charged ions that it produces [18-20]. As a further advantage, the high number of charges of ESI ions of the highest mass values yields mass-to-charge ratio values generally in the range 500-2500, within the mass-to-charge upper limit of most types of mass spectrometers. This has the disadvantage, however, that the mass value must usually be derived from two or more peaks of the same mass value but different charge values; this becomes increasingly difficult for spectra containing an increasing number of mass values and/or decreasing number of charge values, such as the tandem mass spectrum from the dissociation of a large protein ion [19]. However, with unit resolution, the ${ }^{12} \mathrm{C} /{ }^{13} \mathrm{C}$ isotopic peaks provide a direct solution to this problem because the number of peaks in a mass-to-charge unit corresponds to the charge value [20-24]

Although magnetic sector instruments could achieve unit resolution for such large protein ions, the required narrow slits allow transmittance of only a concomitantly small proportion of the large mass range, which must be scanned while consuming the sample in continuous ionization. Array detectors [5] can measure a larger, but still relatively small, part of the spectrum with some sacrifice in resolving power. Further, these 
and most mass spectrometric instruments detect ions by collision with an electron multiplier; measuring ions nondestructively allows them to be remeasured for improved signal-to-noise ratio [25] and also to be dissociated or reacted for MS-II characterization [9], with this repeated for $\mathrm{MS}^{n}$ [26].

An instrument that appears to combine uniquely these attributes for $\mathrm{MS}^{n}$ of large molecules is the Fourier-transform ion cyclotron resonance (ICR) mass spectrometer [26-34]. Details are given here concerning an ESI Fourier-transform mass spectrometry (FTMS) instrument for the MS $^{n}$ characterization of proteins that exhibits greater than $10^{6}$ resolving power and less than 1-ppm mass measurement errors [20].

\section{Experimental}

The basic instrument configuration consists of a modified Millipore Extrel (Madison, WI) FTMS 2000 FT /ICR equipped with a 6.2-T magnet, an Odyssey data system, and external ESI source following the design of the earlier system [21-24]. The electrosprayed ions are conveyed from the source to the trapped-ion analyzer cell by three sequential radiofrequency-only quadrupole lenses through five stages of differential pumping (Figure 1).

The electrospray originates from a 32-gauge syringe needle biased at $2-3 \mathrm{kV}$, to which aqueous protein sample solutions are delivered at a flow rate of 1.5 $\mu \mathrm{L} / \min$ by a Harvard Apparatus (South Natick, MA) syringe pump. The electrosprayed ions enter the first region of differential pumping through a $20-\mathrm{cm}$ long, $0.5-\mathrm{mm}$ inner diameter (i.d.) stainless steel capillary [35]. 'The capillary is resistively heated to temperatures of $100-250^{\circ} \mathrm{C}$, with applied direct currents of $2-6 \mathrm{~A}$ to promote desolvation of ions with no countercurrent gas flow [20, 24, 35]. The exit end of the capillary terminates $7 \mathrm{~mm}$ from a Beam Dynamics skimmer with a $0.5-\mathrm{mm}$ sampling orifice (Minneapolis, MN); the capillary/skimmer region is evacuated to a pressure of 1 torr by a 38-L/s Laybold (Pittsburgh, PA) Sogevac SV280 rotary pump. Further desolvation of ions in this region is accomplished by collisional activation promoted by biasing the capillary at $150 \mathrm{~V}$ with respect to the skimmer, or higher potentials may be used to promote ion dissociation $[18,19]$.

Ions exit the skimmer (which is biased at $30 \mathrm{~V}$ relative to ground) and are conveyed into the Fouriertransform mass spectrometer by the three quadrupole lenses. Each quadrupole is independently evacuated, and conductance limits are located at the exit of each lens to provide three additional stages of differential pumping. Source isolation is provided by a 2.75 in. gate valve located between the first and second quadrupole, allowing source maintenance without venting the high-vacuum regions of the instrument. The first quadrupole is pumped by an $800-\mathrm{L} / \mathrm{s} \mathrm{Ed}$ wards diffusion pump (West Sussex, UK) to a pressure of $10^{-5}$ torr, the second by a $300-\mathrm{L} / \mathrm{s}$ Alcatel diffusion pump (Grand Island, NY) to $10^{-6}$ torr, and the third by a $1500-\mathrm{L} / \mathrm{s}$ Cryo-Torr model 8 cryopump (Helix Technology, Waltham, MA) to $10^{-8}$ torr. Two Extranu-

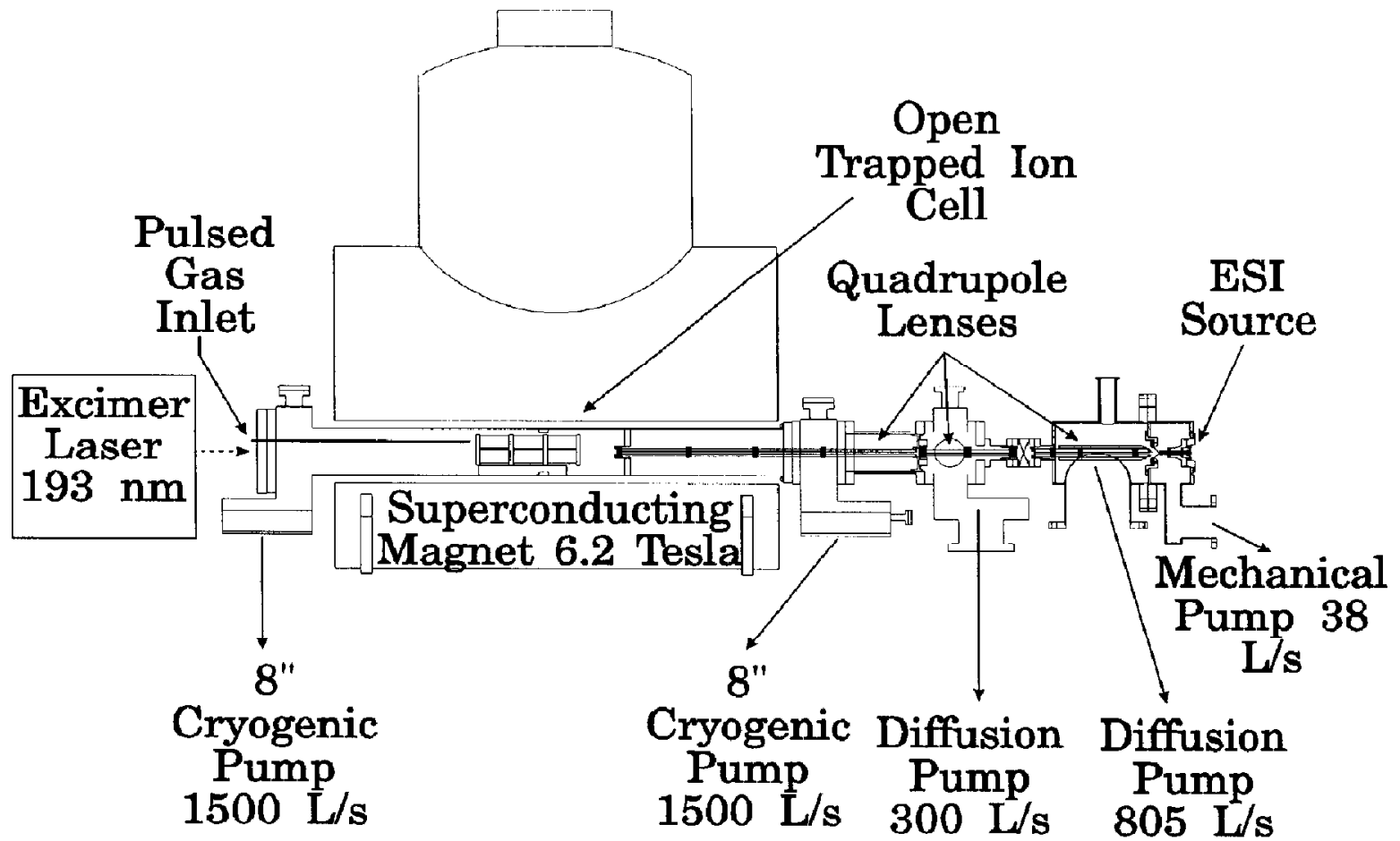

Figure 1. Schematic of new FTMS instrument. 
clear power supplies (Pittsburgh, PA) equipped with High $Q$ heads drive the first quadrupole and the second and third quadrupoles in tandem, respectively. The first driver runs continuously, whereas the second is gated on by the data system only during ion injection events. Each driver may be independently biased to provide acceleration of ions between quadrupoles, with the first and second typically biased at $10-20$ and $-250 \mathrm{~V}$, respectively. The first potential controls the final energy of ions entering the trapped-ion cell because this intralens region is the first in which the pressure is low enough for the ions to gain energy corresponding to the full potential drop between stages. The second potential provides high-energy injection of the ions for efficient penetration of the magnetic field; energy gained in this stage is lost when the ions exit the third lens, and thus its potential has little effect on the final energy of the ions.

Ions exit the third quadrupole well inside the highfield region of the magnet and travel a distance of 10 $\mathrm{cm}$ to the trapped-ion cell. Typical ion currents to the cell are $30-150 \mathrm{pA}$. The cell is an all-copper open cell [36] of cylindrical geometry (5-cm diameter) and a 2:1 aspect ratio. The cell region is evacuated with a second Cryo-Torr model 8 cryopump to provide the fifth and final stage of pumping. Base pressure in the analyzer region is typically less than $2 \times 10^{-9}$ torr, with no measurable increase in pressure during operation of the external ESI source. Static trapping potentials are maintained during ion injection, and pulsed-valve introduction of nitrogen is used to effect collisional deceleration and retention of ions [22 24]. Because the pulse valves would not operate properly in the strong magnetic field, they are placed approximately $70 \mathrm{~cm}$ from the bore of the magnet, and a 3/8-in. i.d. stainless steel tube is used to conduct the gas to within $2 \mathrm{~cm}$ of the trapped-ion cell. Peak pressure during the trapping event is typically $5 \times 10^{-6}$ torr, and analyzer base pressure is restored in $20-30 \mathrm{~s}$. Amplification of the $0-10-V$ trap driver in the Odyssey cell controller allows potentials up to $30 \mathrm{~V}$ to be used for accumulation of higher energy ions.

Ion radial excitation is typically accomplished with a broadband frequency sweep, and the resulting transients are digitized at up to $2 \mathrm{M}$ data points per spectrum. This large memory capacity allows 6-s acquisitions at a bandwidth of $150 \mathrm{kHz}$, corresponding to a lower mass limit of $m / z$ 600. Standard heterodyne techniques [34] were used to acquire for longer periods for ultrahigh-resolution measurements.

All compounds were obtained from Sigma Chemical Co. (St. Louis, MO) and used without further purification. Equine cytochrome $c(12.3 \mathrm{kDa})$ was used for all ion injection studies unless otherwise noted.

\section{Ion Injection}

The ion generation and injection process results in some residual axial ion kinetic energy on arrival at the cell. It is important that this energy be known and minimized to design effective trapping techniques. Retarding potential studies of an injected ion beam comprising the full charge-state envelope of cytochrome $c$ ions $(+16$ to +7$)$ with a $20-V$ offset on the first quadrupole lens indicate that the ion energy distribution is approximately Gaussian, with a maximum at approximately $15 \mathrm{eV}$ per charge and a full width at half maximum of approximately $12 \mathrm{eV}$ per charge. Similarly, the average energy per charge is always somewhat less than the quadrupole potential offset at both higher and lower voltages, consistent with collisional retardation in this approximately $10^{-3}$-torr region. This relatively wide apparent kinetic energy per unit charge distribution, as determined by a retarding potential study, may be attributed to a variety of effects. For example, if all of the ions from the +16 to +7 charge-state envelope of cytochrome $c$ are accelerated to the same velocity of $1000 \mathrm{~m} / \mathrm{s}$ in the supersonic expansion of the ESI source, then the retarding potential profile would extend from 4 to $9 \mathrm{~V}$, even though all of the ions have the same kinetic energy. This is a simple consequence of the fact that the retarding force experienced by the ions is proportional to the charge on the ion. Convoluting the energy distribution that results from electrostatic acceleration in a highpressure region with the wide distribution of ion charge states and the distribution of velocities gained in the supersonic expansion yields a wide apparent distribution of ion energies. Additional contributions to the distribution are encountered on injecting ions with a distribution of radial energies acquired in the quadrupole lenses, through the strong magnetic field gradient of the superconducting magnet (the wellknown magnetic mirror effect). Because the width of the kinetic energy per unit charge distribution can have an important effect on overall trapping efficiency (see lon Trapping), there is a continuing effort to find a combination of source and injection parameters that will minimize this distribution. To facilitate trapping for a given distribution width, it is similarly important to minimize the energy per charge corresponding to the distribution maximum, a parameter principally controlled in this instrument by the offset potential on the first quadrupole. A higher offset potential on this element yields more efficient ion injection; however, in practice an offset of 10-20 V results in the best compromise between high-efficiency injection and low residual ion energy.

\section{Ion Trapping}

Two approaches are common for collecting externally generated ions in the cell-gated and accumulated trapping [37]. Gated trapping involves lowering the applied potential at either end of the cell to allow ions into the cell and then raising the potential to retain the ions. This technique requires that the ion energy be lower than the available trapping potentials and is 
most effective for pulsed-ion production. For our continuous-ionization instrument, accumulated trapping, such as used by Laude and co-workers [37, 38], has been much more effective. Static trapping potentials are maintained while injecting ions with kinetic energy sufficient to penetrate the trapping field; deceleration in the cell traps the ions between the static potentials. The most likely deceleration mechanism is collision with neutral gas molecules, which has proved effective in higher pressure external source instruments [38]. In fact, at pressures lower than approximately $10^{-8}$ torr, accumulation is essentially undetectable. Determination of total accumulated ion abundance as a function of static analyzer pressure (from leak-valve introduction of nitrogen) indicates that accumulation increases dramatically with pressure up to $10^{-6}$ torr. Beyond this range, collisional scattering, space-charge effects, and other losses apparently offset improved trapping. Such effects can be minimized by providing high pressures only during the ion injection event. Thus, pulsed-gas introduction during ion injection proved to be a convenient way to increase the trapping efficiency; peak pulse pressures of approximately $10^{-5}$ torr with injection times of less than $1 \mathrm{~s}$ require approximately $20 \mathrm{~s}$ to restore the base pressure required for highperformance excitation and detection of the acquired ions. Although sufficient ion populations are easily acquired with this technique, overall trapping efficiency is quite low. Using a statistical method to estimate the number of ions in the cell [39], a typical trapping efficiency of only $0.1 \%$ is calculated based on the measured cell current and ion injection period.

Consistent with the results of Hofstadler and Laude [38], the effect of trapping potential on total ions accumulated generally mimics the kinetic energy profile of the injected ion population. Because the ions are required to traverse the $10-\mathrm{cm}$ distance between the injection quadrupole and cell during the high-pressure injection event, some ion deceleration occurs prior to arrival at the cell, and thus the optimum trapping potential may be as much as a few volts lower (depending on the pressure used for trapping) than would be indicated by the energy distribution measured at low pressure. Although relatively high trapping potentials are required for optimum accumulation, most of the ion kinetic energy was damped sufficiently within $1 \mathrm{~s}$ for the ions to be retained with much lower trapping potentials (typically $\leq 1 \mathrm{~V}$ ). Thus, following ion injection, the potential is stepped down to this range to minimize trapping potential-induced frequency shifts and to promote optimum ion detection. Experiments with ramped-down rather than stepped changes provided no improvement in subsequent detection, and thus the simpler stepped change is used in this work.

As was observed with Laude and co-workers' [38, 40] internal electrospray instrument, the efficiency of acquisition with this instrument also varies with charge state as well as with trapping potential. The total ion kinetic energy is the sum of a component from the supersonic expansion in the source, in which each ion is accelerated to the same velocity and energy, dependent on mass and collision cross section but independent of charge, and an electrostatic component from acceleration between lens elements of different potential to add a constant energy per unit charge. Thus, the total energy per unit charge varies, and the extent of this determines the variation in optimum trapping potential per number of charges. For example, a more highly charged ion will require a lower trapping potential than a less charged ion of the same kinetic energy because the force experienced by an ion in a given electric field is proportional to the charge on the ion. These effects have recently been explored in some detail, and the mass-to-charge discrimination that results from such effects has been demonstrated to provide a method for selective mass and charge-state acquisition of electrosprayed ions [40]. Such capability appears to be a general feature of the ESI/FT/MS experiment, independent of the manner in which ions are introduced to the cell.

The extent of initial ion deceleration during the trapping process may be probed by varying the potential difference between the rear and front trap plates (Figure 2). Ions with sufficient energy to penetrate the first trap must lose enough kinetic energy during their passage through the cell to be retained by the rear trap
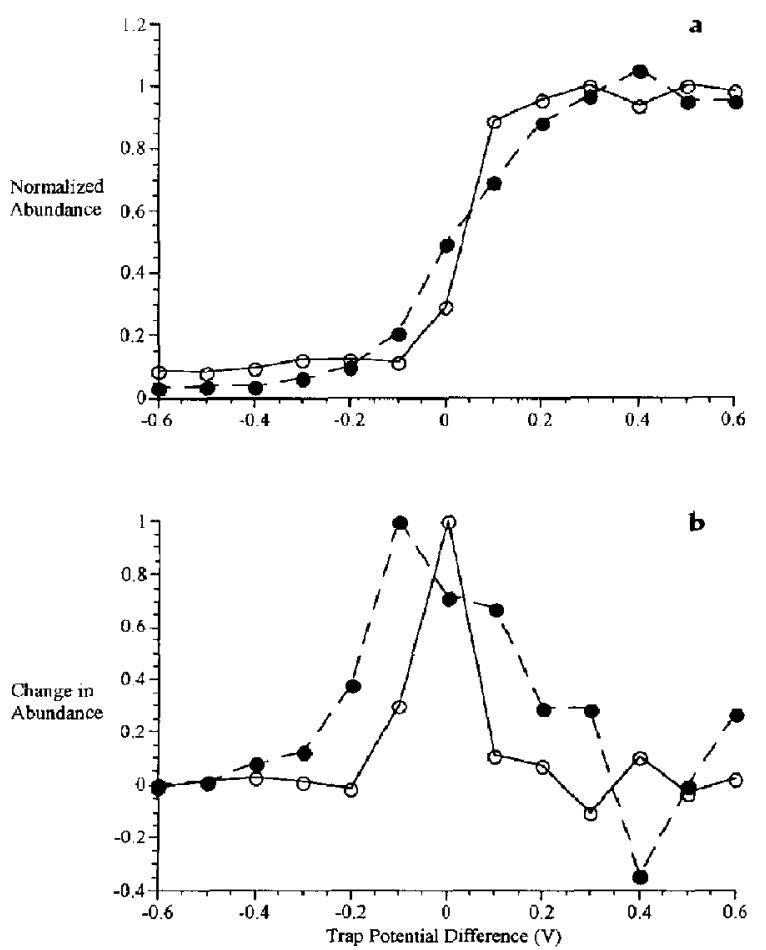

Figure 2. (a) Trapped ions accumulated as a function of trap plate potential difference, static cell pressure: (O) $3 \times 10^{-6}$ torr; (O) $6 \times 10^{-6}$ torr. (b) Differential of trap plate potential difference profile shown in $\mathbf{a}$. 
potential. The steeper curve at the lower pressure in Figure $2 \mathrm{a}$ is consistent with less (and a narrower range of) ion energy lost during the initial cell traversal, as expected. This is shown more clearly in differential form in Figure $2 b$; minimizing the width of the final axial kinetic energy distribution of the acquired ions has been found to promote high-resolution measurements. However, even at the higher pressure, it is apparent that the accumulated trapping technique retains a relatively narrow "slice" ( $<0.5 \mathrm{eV}$ per charge) of the injected kinetic energy per unit charge distribution. It is for this reason that it is desirable to minimize the width of that distribution and thus maximize the population density within the acquired energy interval.

\section{High Resolution}

It is well known [31-33] that the higher field magnet and lower system pressure from the larger capacity pumping system should improve performance; however, factors that affect transient lifetimes and field homogeneities are also important. The support structure for the original trapped-ion cell exhibited an unusually large magnetic susceptibility, so this was replaced with a laboratory-built copper open cell [36]. The open geometry" is inherently more compatible with external ion injection, as well as providing lower electric field inhomogeneities. It was also found that cell and cell lead vibrations, as well as low-frequency noise in the detection circuitry, resulted in reduced ion storage time and shorter transients; such excitation of low-frequency (trapping and magnetron) modes could promote ion cloud expansion and ion loss. Finally, low trap potentials ( $\leq 1 \mathrm{~V}$ ), ion cooling delays (usually 120 s), and small ion populations were found to improve transient lifetimes significantly. Space-charge effects imposed by the density of the ion population are perhaps the most significant factor and require a careful compromise between the objectives of high signalto-noise ratio and high resolution to achieve spectra (Figures 3 and 4) of 8.6-29-kDa proteins of 2-0.5 $\times 10^{6}$ resolving power.

Figures 3 and 4 show three examples of high-resolution spectra acquired with the new instrument. Figure 3a is a high-resolution spectrum of bovine ubiquitin $(8565 \mathrm{Da})$, with a resolving power of $2 \times 10^{6}$. This spectrum was collected with a 0.5 -s ion beam injection period ( $250 \mathrm{fmol}$ injected), with 13 and $14 \mathrm{~V}$ on the front and rear trap plates, respectively, during the beam event. The trap potentials were then decreased to $1 \mathrm{~V}$, and the ions were cooled for $120 \mathrm{~s}$. Excitation was then performed using a chirp from 50 to $150 \mathrm{kHz}$ at a sweep rate of $100 \mathrm{~Hz} / \mu \mathrm{s} ; 2 \mathrm{M}$ data points were then collected in heterodyne mode at a bandwidth of $35 \mathrm{kHz}$, corresponding to $m / z 770-1075$. (Although the signal acquisition period was $30 \mathrm{~s}$, the transient lifetime in this example was only approximately $20 \mathrm{~s}$, and thus resolution is not data-point limited.) The
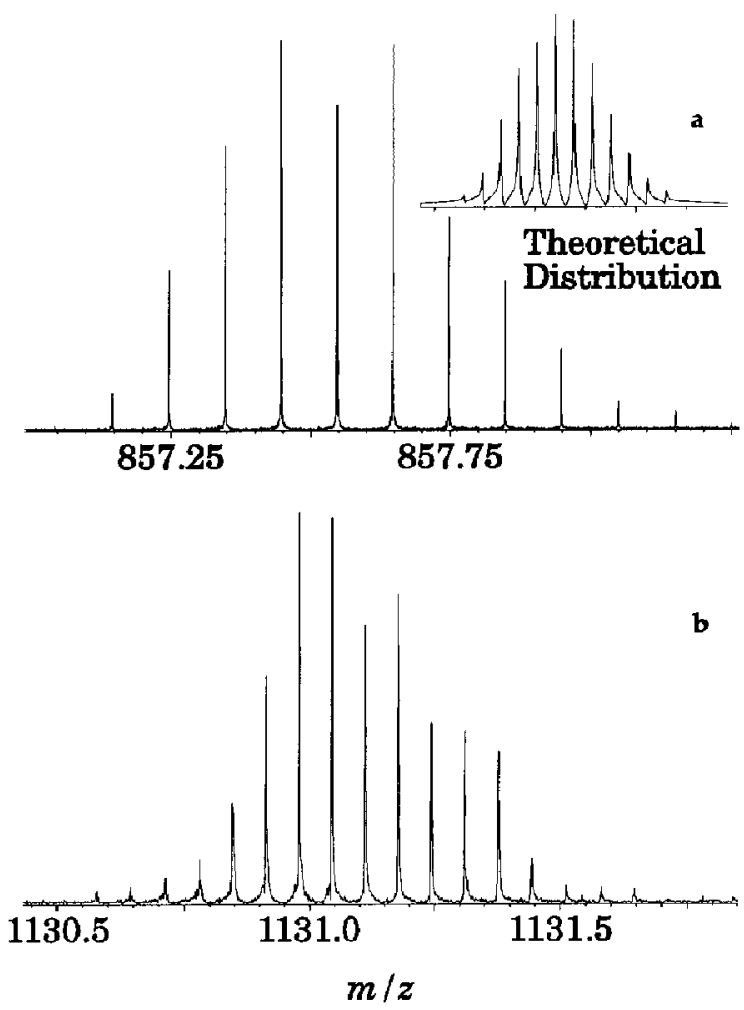

Figure 3. Single-scan partial mass spectrum of (a) ubiquitin $(8565 \mathrm{Da})$, resolving power $2 \times 10^{6}$, and (b) apomyoglobin $(16,952$ Da), resolving power $9 \times 10^{5}$.

central peak in the isotopic cluster appears smaller than the adjacent peaks because the two highest points on the peak are at the same abundance and by chance symmetrically disposed about the peak maximum, making the peak appear flat-topped. Zero filling the data before processing would improve the appearance of the isotopic profile, but this is not possible on the

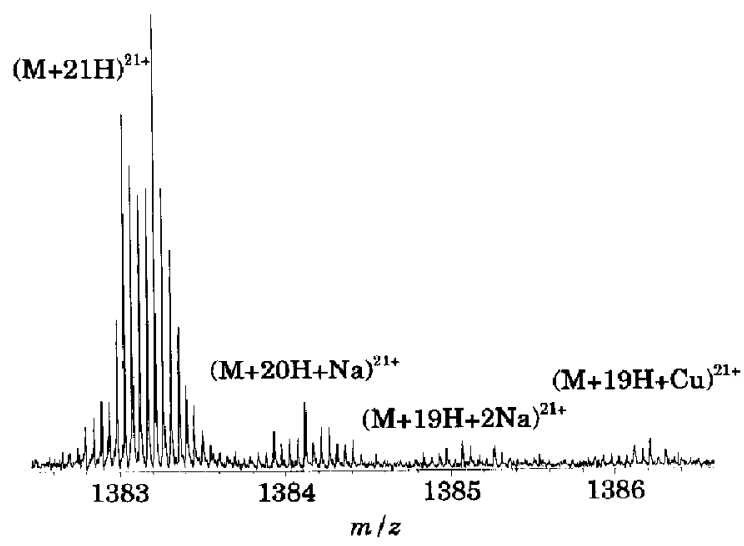

Figure 4. Single-scan partial mass spectrum of carbonic anhydrase $(29,025 \mathrm{Da})$, resolving power $5 \times 10^{5}$. 
current data system for a $2 \mathrm{M}$ point spectra owing to memory limitations.

Figure $3 \mathrm{~b}$ is a comparable spectrum of horse skeletal muscle apomyoglobin $(16,952 \mathrm{Da})$ recorded at $9 \times$ $10^{5}$ resolving power. The conditions were the same as in Figure 3a, except that a 1-s beam was used $(0.5$ pmol), with a $75-\mathrm{Hz} / \mu$ s sweep rate and a bandwidth of $79 \mathrm{kHz}$, covering the range $m / z \quad 750-2000$. The irregular shape of the isotopic envelope is attributed to small population ion statistics. With at most a few thousand ions contributing to the entire isotopic profile, there will be significant random fluctuations from the theoretical abundances.

Figure 4 is a spectrum of bovine carbonic anhydrase II $(29,025 \mathrm{Da})$ recorded at a resolving power of $5 \times 10^{5}$. Experimental conditions were the same as in Figure 3, except that a $2-s$ beam was used ( 1 pmol), and only $1 \mathrm{M}$ data points were collected, thus making the resolving power data-point limited. With this signal-to-noise level, both sodium and copper adducts are visible in the spectrum. These adduct assignments are confirmed by accurate mass measurements and by doping the samples with the corresponding acetate salt, which increases the abundance of the corresponding adduct peaks. The copper ions result from the reaction of acidic sample solutions with exposed brass in the nickel-plated brass fittings of the sample syringe [20].

The effect of static system pressure (nitrogen) on resolution was determined for spectra of cytochrome $c$. Isotopic resolution is lost at $6 \times 10^{-8}$ torr, although with $\mathrm{D}_{2} \mathrm{O}$, a lower mass gaseous neutral, isotopic resolution can still be obtained at $10^{-7}$ torr (Tigure 5) [24].

Theory predicts that resolving power should decrease with increasing mass-to-charge ratio, even for ions of the same mass value, when not under pressure-limited conditions. Figure 6 shows the resolving power measured for cytochrome $c$ versus massto-charge (closed circles), whereas the line represents the predicted reciprocal relationship. This gratifying agreement is in contrast to recent higher pressure measurements of Hofstadler and Laude [38] in which the isotope peaks were not resolved, so that observed peak widths are dominated by the width of isotope distribution profiles. In fact, only under high-pressure (10 $0^{-6}$-torr range) conditions is the resolving power observed to increase rather than decrease with increasing mass-to-charge for ions of the same mass. This increase is linear with mass-to-charge, consistent with resolution decreased by collisional damping, with the collision rate determined by ion velocity, which decreases linearly with increasing mass-to-charge.

It is important to note that although long transient lifetimes are a prerequisite for obtaining high resolution, such lifetimes alone are not a sufficient condition. If during the evolution of the transient the ions experience a variation in the magnetic field or nonquadrupolar variation in the electric field, there will be a corresponding shift in the effective cyclotron frequency during the transient. Such a continuous frequency shift

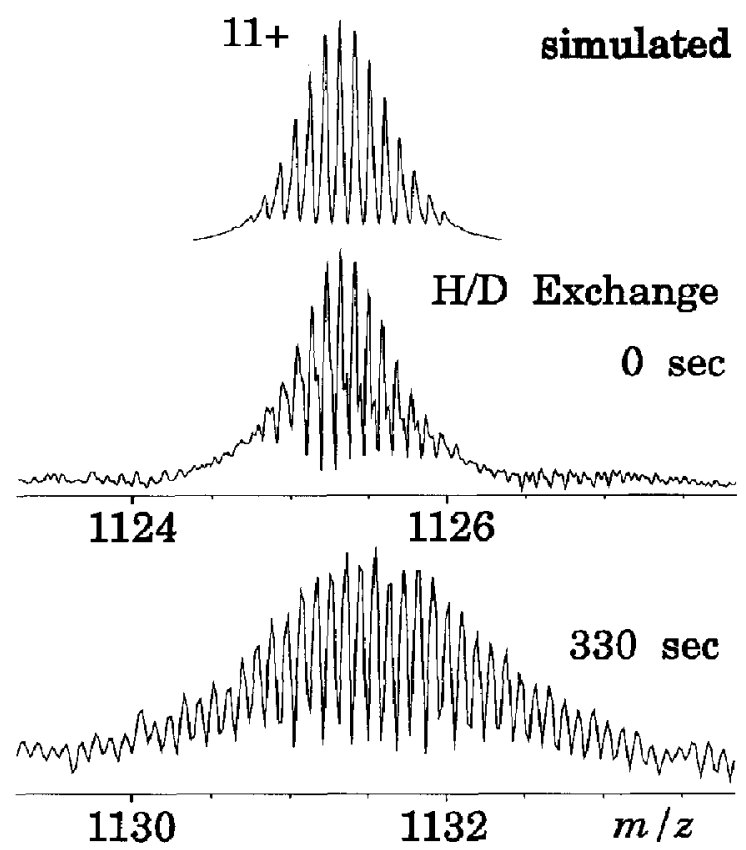

Figure 5. Effect of $1 \times 10^{-7}$ torr of $D_{2} O$ on the resolution of cytochrome $c$ with $\mathrm{H} / \mathrm{D}$ exchange.

will cause a corresponding broadening of peaks. To the extent that the transient decay of such large ions represents a corresponding decay in the radial amplitude of ion motion [25] rather than a dephasing of coherent ion motion, radial field inhomogeneities should be the primary cause of frequency shifts during the transient. Radial magnetic field inhomogeneities may exist owing to intrinsic problems with the magnet, nonoptimum positioning of the cell, or the use of highsusceptibility materials for cell or cell support construction. Nonquadrupolar electric fields are likely to be experienced whenever the radial amplitude of ion motion exceeds about one half of the cell radius or

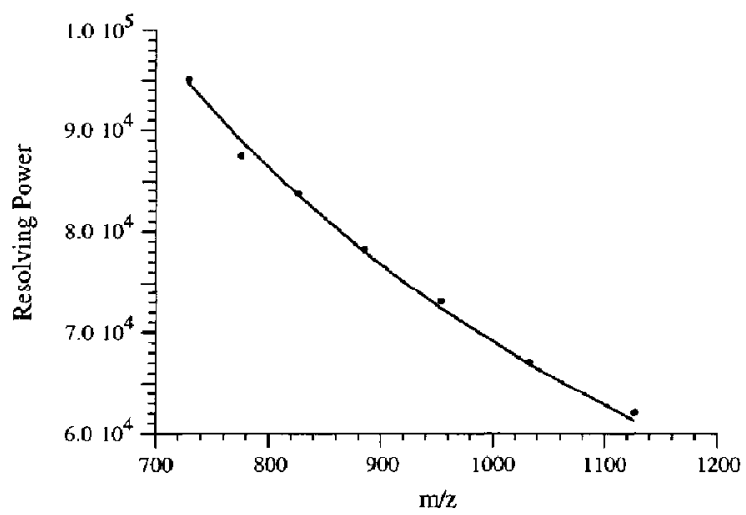

Figure 6. Effect of mass-to-charge ratio value on resolving power for cytochrome $c(12,361 \mathrm{Da})$ isotopic peaks: (O) measured valves; ( - ) theoretically predicted function. The letters indicate the amono acid composition of fragments. 
when ion population densities are large enough to cause significant Coulombic interaction between ions. Regardless of the cause, such frequency shifts will be evidenced by a frequency resolution that does not correspond to the inverse of transient lifetime; the highest resolution may then be obtained for observation times significantly shorter than the transient decay time. This effect may be used as a simple diagnostic tool for probing field inhomogeneities in the trappedion cell.

Although these advances in high resolution allow mass determinations with errors of less than $0.1 \mathrm{Da}$, there is still the potential to make an error of $\pm 1 \mathrm{Da}$ from an incorrect assignment for the number of heavy isotopes present in a specific peak. This is not a problem for smaller ions because the peak of lowest mass must be monoisotopic; however, its intensity decreases as mass increases because of the increased probability for multiple heavy isotopes. For ubiquitin, the monoisotopic peak abundance is less than $4 \%$ of that of the most abundant peak (Figure 3a). The monoisotopic mass of a molecule can be estimated using the average molecular weight from the unresolved isotopic cluster [41].

For resolved isotopic peaks, their measured intensity profile can be compared with a model theoretical profile to assist in the assignment for the monoisotopic mass. The profile expected as a function of protein molecular weight was derived from the statistical oc currence of elemental composition of the amino acids from the Protein Identification Resource (National Biomedical Research Foundation, Washington, DC) protein data base. Using the measured average molecular weight of a compound, the theoretical isotopic profile is calculated [42]. Tu improve the precision of abundance values, isotopic profiles from multiple charge states are convoluted [43]. Details of the method and its successful routine application are reported elsewhere [39].

This instrument configuration with its unusual capabilities for high-resolution measurements of large ions has several potential advantages. These include determination of the charge state of unknown ions based on ${ }^{13} \mathrm{C}$ isotopic spacing [21-23], observation of low-mass adducts on large molecules (Figure 4), improved tracking of isotope-exchange reactions [24], improved mass accuracy ( $<1 \mathrm{ppm}$ ) for isotopically resolved peaks [20], and distinguishing isobaric elemental composition assignments (glutamine and lysine contribute 128.058 and $128.095 \mathrm{Da}$, respectively, to the protein mass, so that 1-ppm mass accuracy will distinguish these at $20 \mathrm{kDa}$ ).

\section{Ion Dissociation}

Further characterization of MS-I-analyzed ions from ESI requires an effective ability for their dissociation and/or reaction. Of our initial dissociation studies, collisionally activated dissociation (CAD) of the radi- ally excited ions was not found to be effective for peptides larger than gramicidin $S(1.2 \mathrm{kDa})$ despite success in triple quadrupole CAD experiments with 66-kDa albumin ions [19]. Photodissociation using a 193-nm laser (Figure 1) is more effective for gramicidin $S$ (Figure 7 ) and 8.6-kDa ubiquitin (Figure 8) but produces predominantly small, less informative fragments that are difficult to assign because of apparent internal fragmentations. Photodissociation of larger ions did not yield observable products, even though the parent ion abundance decreased with increasing irradiance. Attempts to trap the presumably high kinetic energy fragments using higher trap potentials and/or pulsedgas collisional cooling were unsuccessful. By far the best dissociation results were obtained with nozzle/skimmer dissociation in the external ion source [18-20], as demonstrated (Table 1) for ubiquitin (Figure 9) and carbonic anhydrase (Figure 10). Here some of the lower abundance fragments of ubiquitin exhibit peak splitting that resembles an overlapping isotope distribution. This splitting is eliminated when smaller ion populations are used (for example, by ejecting higher abundance ions) and thus is apparently caused by space-charge effects. For carbonic anhydrase, the masses of 38 fragment ions could be assigned directly from their isotopic spacing, with 15 of these corresponding to terminal fragments of the known sequence. Nozzle/skimmer dissociation thus appears to bc quite effective in dissociating larger ions and further provides abundant large-fragment ions $(5-10 \mathrm{kDa})$ that may prove to be amenable to further dissociation in the trapped-ion cell by conventional $C A D$ or photodissociation techniques. A disadvantage of the nozzle/skimmer technique is that it indiscriminately dissociates all electrosprayed ions, forfeiting the significant MS/MS advantage of selectivity.

The trapped-ion capabilities of FTMS also allow characterization of the mass-analyzed primary ions by ion-molecule reactions. For example, $H / D$ exchange

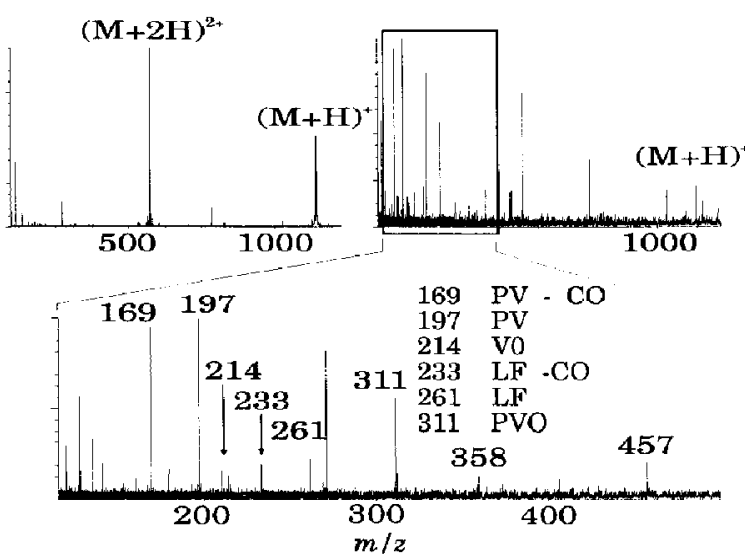

Figure 7. Photodissociation $(193 \mathrm{~nm})$ of gramicidin $S$ ions before and after 25 laser shots. The letters indicate the amino acid composition of fragments. 


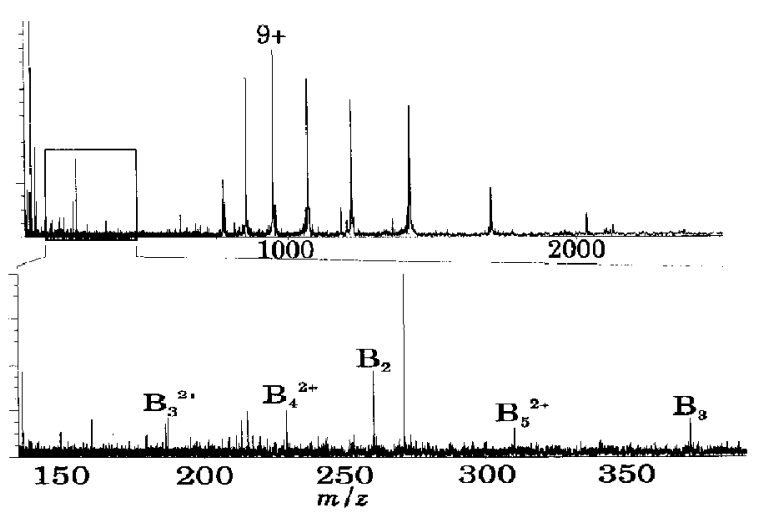

Figure 8. Photodissociation (193 nm) of ubiquitin ions (8565 Da), 10 scans.

Table 1. Assigned dissociation fragments for ubiquitin and carbonic anhydrase

\begin{tabular}{cll}
\hline Fragment & Charge & \multicolumn{1}{c}{$\mathrm{m} / \mathrm{z}$} \\
\hline \hline Ubiquitin & & \\
$V_{24}$ & 4,3 & 683,910 \\
$V_{18}$ & 3,2 & 700,1050 \\
$Y_{58}$ & $9,8,7$ & $727,818,935$ \\
$b_{16}$ & 2 & 904 \\
$b_{18}$ & 2 & 1018 \\
Carbonic & & \\
anhydrase & & \\
$V_{24}$ & 4 & 741 \\
$b_{31}$ & 4 & 912 \\
$b_{40}$ & 5 & 924 \\
$V_{67}$ & $8,7,6$ & $951,1087,1268$ \\
$V_{25}$ & 3 & 987 \\
$Y_{61}$ & $7,6.5$ & $1007,1175,1410$ \\
$b_{135}$ & 15 & 1022 \\
$V_{78}$ & 8,7 & 1095,1251 \\
$Y_{63}$ & 6 & 1208 \\
$Y_{68}$ & 6 & 1295 \\
\hline
\end{tabular}

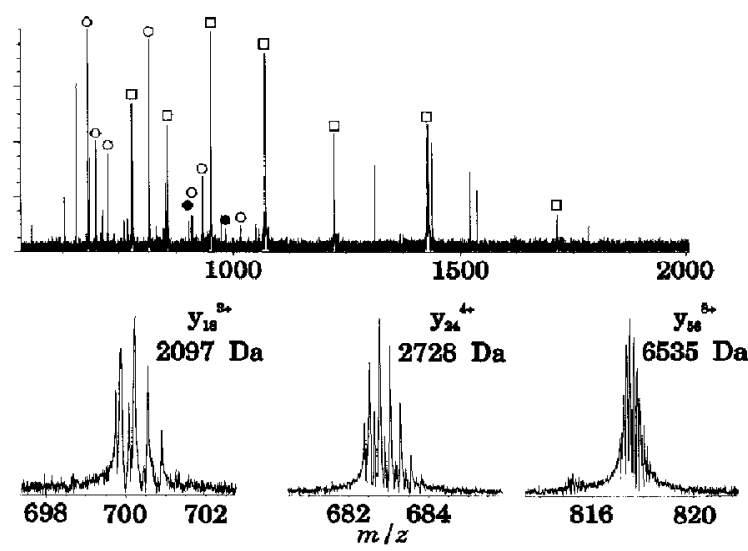

Figure 9. Nozzle/skimmer $(150 \mathrm{~V})$ dissociation of ubiquitin ions: (O) y-type ions; ( ) b-type ions; ( $\square$ ) molecular ions.

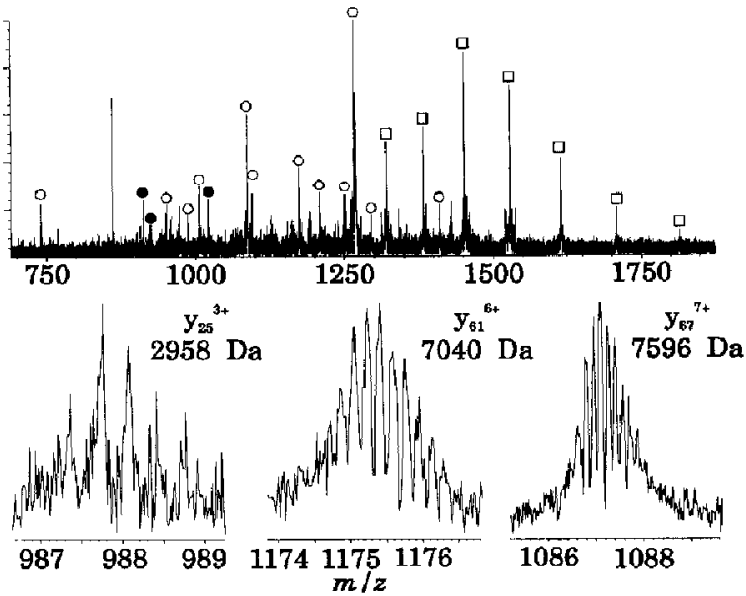

Figure 10. Nozzle/skimmer dissociation of carbonic anhydrase ions $(29.0 \mathrm{kDa})$; symbols as in Figure 9.

with multiply charged protein ions by $\mathrm{D}_{2} \mathrm{O}, \mathrm{CH}_{3} \mathrm{OD}$, $\mathrm{CH}_{3} \mathrm{COOD}$, and $\mathrm{ND}_{3}$ have demonstrated the stability of multiple three-dimensional conformers [24].

\section{Conclusions}

The triple quadrupole $[6,44]$ and the tandem doublefocusing magnetic sector instruments $[4,5,45]$ are now recognized as unique tools for providing definitive molecular weight and sequence information on oligopeptides $(<3 \mathrm{kDa})$, even in subpicomole amounts and as components of mixtures. The FTMS instrument described here could be the forerunner of a new generation of mass spectrometers, providing such information on biomolecules larger by one or even two orders of magnitude and using samples smaller by even three orders of magnitude.

\section{Acknowledgments}

We are grateful to J. E. Campana, J. B. Fenn, M. L. Gross, D. F. Hunt, J. A. Loo, J. Shabanowitz, A. Slapikas, B. H. Wang, C. M. Whitehouse, C. L. Wilkins, E. R. Williams, and M.-Y. Zhang for valuable help.

We thank the National Institutes of Health (Grant GM16609) and the Gavlin Foundation for generous financial support.

\section{References}

1. Biemann, K. Mass Spectrometry: Organic Chemical Applications; McGraw-Hill: New York, 1962; pp 283-296.

2. Senn, M.; Venkataraghavan, R.; McLafferty, F. W. I. Am. Chem. Soc. 1966, 88, 5593.

3. Biemann, K.; Cone, C.; Webster, B.; Arsenault, G. P. I. Am. Chem. Soc. 1966, 88, 5598.

4. Biemann, K. Biomed. Environ. Mass Spectrom. 1988, 16, 99-111.

5. Carr, S. A.; Hemling, M. E.; Bean, M. F.; Roberts, G. D. Anal. Chem. 1991, 63, 2802-2824. 
6. Hunt, D. F; Michel, H.; Dickenson, T. A.; Shabanowitz, J.; Cox, A. L.; Sakaguchi, K.; Appella, E.; Gray, H. M.; Sette, A. Science 1992, 256, 1817-1820.

7. McLafferty, F. W; Venkataraghavan, R.; Irving, P. Biochem. Biophys. Res. Commun. 1970, 39, 274.

8. Wipf, H.-K.; Irving, P.; McCamish, M.; Venkataraghavan, R.; McLafferty, F. W. J. Am. Chem. Soc. 1973, 95, 3369.

9. Cody, R. B. Jr.; Amster, I. J.; McLafferty, F. W. Proc. Nati. Acad. Sci. USA 1985, 82, 6367-6370.

10. Levsen, Ka; Wipf, H.-K.; McLafferty, F. W. Org. Mass Spectrom. 1974, 8, 117.

11. Macfarlane, R. D.; Hill, J. C.; Jacobs, D. L.; Phelps, R. G. In Mass Spectrometry in the Analysis of Large Molecules; MeNeal, Ed.; John Wiley: New York, 1986; pp 1-12.

12. Barber, M.; Bordoli, R. S.; Sedgwick, R. D.; Tyler, A. N. J. Chem. Soc., Chem. Commun. 1981, 3, 25.

13. Hillenkamp, F.; Karas, M.; Beavis, R. C.; Chait, B. T. Anal. Chem. 1991, 63, 1193A-1202A.

14. Clegg, G. A.; Dole, M. Biopolymers 1971, 10, 821-826.

15. Fenn, J. B.; Mann, M.; Meng, C. K.; Wong S. F.; Whilehouse, C. M. Science 1989, 246, 64-71.

16. (a) Smith, R. D.; Loo, J. A.; Ogorzalek Loo, R. R.; Busman, M.; Udseth, H. R. Mass Spectrom. Rev. 1991, 10, 359-451; (b) Smith, R. D.; Loo, J. A.; Ogorzalek Loo, R. R.; Busman, M.; Udseth, H. R. Mass Spectrom. Rev. 1992, 11, 434-443.

17. Griffin, L. L.; McAdoo, D. J. J. Am. Soc. Mass. Spectrom. 1993, $4,11-15$.

18. Loo, J. A.; Udseth, H. R.; Smith, R. D. Rapid Commun. Mass Spectrom. 1988, 2, 207-210.

19. Loo, J. A., Edmonds, C. G.; Smilh, R. D. Anal. Chem. 1991, 63, 2488-2499.

20. Beu, S. C.; Senko, M. W.; Quinn, J. P.; McLafferty, F. W. J. Am. Soc. Mass Spectrom. 1993, 4, 190-192.

21. Henry, K. D.; McLafferty, F. W. Org. Mass Spectrom. 1990, 25, 490-492.

22. Henry, K. D.; Quinn, J. P.; Mclafferty, F. W. I. Am. Chem. Soc. 1991, 113, 5447-5449.

23. Loo, J. A.; Quinn, J. P.; Ryu, S. I.; Henry, K. D.; Senko, M. W.; McLafferty, F. W. Proc, Natl. Acad. Sci. USA 1992, 89, 286-289.

24. Suckau, D.; Shi, Y.; Beu, S. C.; Senko, M. W.; Quinn, J. P.;
Wampler, F. M. III; McLafferty, F. W. Proc. Natl. Acad. Sci. USA 1993, 90, 790-793.

25. Williams, E. R.; Henry, K. D.; McLafferty, F. W. J. Am. Chem. Soc. 1990, 112, 6157-6162.

26. Freiser, B. S.; Jacobson, D. B. Science 1984, 112 (cover page).

27. Comisarow, M. B.; Marshall, A. G. Chem. Phys. Lett. 1974, 25, $282-283$.

28. White, R. L.; Ledford, E. B.; Ghaderi, S.; Wilkins, C. L.; Gross, M. L. Anal. Chem. 1980, 52, 252.

29. Castro, M. E.; Russell, D. H. Anal. Chem. 1984, 56, 578.

30. Cody, R. B. Jr.; Kinsinger, J. A.; Ghaderi, S., Amster, I. J.; McLafferty, F. W.; Brown, C. E. Anal. Chim. Acta 1985, 178, 43-66.

31. Buchanan, M. V., Ed. Fourier Transform Mass Spectrometry; American Chemical Society: Washington, D.C., 1987.

32. Wilkins, C. L.; Chowdhury, A. K.; Neuwaysir, L. M.; Coates, M. L. Mass Spectrom. Rev. 1989, 8, 67-92.

33. Marshall, A. G; Grosshans, T. B. Anal. Chem. 1992, 63, 215A-229A.

34. Marshall, A. G., Schweikard, L. Int. T. Muss Spectrom. Ion Processes 1992, 118,119, 37-70.

35. Chowdury, S. K.; Katta, V.; Chait, B. T. Rapid Commun. Mass Spectrom. 1990, 4, 81-87.

36. Beu, S. C.; Laude, D. A. Jr. Int. J. Mass Spectrom. Ion Processes $1992,112,215-230$.

37. Beu, S. C.; Laude, D. A. Jr. Int. J. Mass Spectrom. Ion Processes $1992,104,109-127$.

38. Hofstadler, S. A.; Laude, D. A. Jr. J. Am. Soc. Mass Spectrom. 1992, 3, 615-623.

39. Senko, M. W, Beu, S. C.; McLafferty, F. W., to be submitted.

40. Hofstadler, S. A.; Beu, S. C.; Laude, D. A. Jr. Anal. Chem. 1993, 65, 312-316.

41. Zubarev, R.; Bonarenko, P. Rapid Commun. Mass Spectrom. $1991,5,276-277$.

42. Yergey, J. Int. J. Mass Spectrom. Ion Phys. 1983, 52, 337-349.

43. Labowsky, M.; Whitehouse, C.; Fenn, J. B. Rapid Commun. Mass Spectrom. 1993, 7, 71-84.

44. Yost, R. A.; Enke, C. G. In Tandem Mass Spectrometry; McLafferty, F. W., Ed.; John Wiley: New York, 1983; pp 175-195.

45. McLafferty, F. W.; Todd, P. J.; McGilvery, D. C:; Baldwin, M. A. I. Am. Chem. Soc. 1980, 102, 3360-3363. 